\title{
Spatial Correlation for DoA Characterization Using Von Mises, Cosine, and Gaussian Distributions
}

\author{
Wamberto J. L. Queiroz, ${ }^{1}$ Francisco Madeiro, ${ }^{2}$ Waslon Terllizzie A. Lopes, ${ }^{1}$ \\ and Marcelo S. Alencar ${ }^{1}$ \\ ${ }^{1}$ Departamento de Engenharia Elétrica, Universidade Federal de Campina Grande, 58.429-900 Campina Grande, PB, Brazil \\ ${ }^{2}$ Escola Politécnica de Pernambuco, Universidade de Pernambuco, 50.750-470 Recife, PE, Brazil
}

Correspondence should be addressed to Waslon Terllizzie A. Lopes, waslon@ieee.org

Received 2 May 2011; Accepted 2 July 2011

Academic Editor: Hoi Shun Lui

Copyright ( 2011 Wamberto J. L. Queiroz et al. This is an open access article distributed under the Creative Commons Attribution License, which permits unrestricted use, distribution, and reproduction in any medium, provided the original work is properly cited.

\begin{abstract}
This paper presents mathematical expressions for the spatial correlation between elements of linear and circular antenna arrays, considering cosine, Gaussian, and Von Mises distributions, for the direction of arrival (DoA) of the electromagnetic waves at the receiver antenna. The expressions obtained for the Von Mises distribution can include or not the mutual coupling effect between the elements and are simpler than those obtained for the cosine and the Gaussian distributions of the angle of arrival. The Von Mises distribution produces spatial correlation expressions in terms of Bessel and trigonometric functions. An exact expression for the spatial correlation, taking into account the mutual coupling, for the circular and linear arrays and an arbitrary number of elements are presented. It can be verified, by numerical evaluation of the expressions, that the coupling between the elements correlates the electromagnetic field, and a separation of half wavelength could not be enough to decorrelate them.
\end{abstract}

\section{Introduction}

The design of modern communication systems usually requires the statistical characterization of parameters, such as the direction of arrival (DoA) of the electromagnetic wave that reaches the receiver antenna. The knowledge of that parameter is valuable when the target is to limit the effects of interference as well as the gain for undesirable signals [1].

The estimation of DoA has been treated by different authors [1] considering the signal samples captured in the equally spaced elements of antenna arrays. This relevant problem has been addressed in many aspects. A general approach is to consider elements with arbitrary directional characteristics in environments corrupted by noise and interference, characterized by arbitrary covariance matrices. As an example, in [2], the author addresses the spatial processing of signals with respect to the multiplicity of transmitters and presents the algorithm used in the multiple signal classification (MUSIC) method which gives asymptotically nonbiased estimates of different parameters, such as number of arriving waves, direction of arrival, interference, and noise power. A comparative study of methods based on maximum likelihood (ML) and maximum entropy (ME) is presented. The approach presented in the paper for the classification of multiple signals is general and has wide application. The method may be understood in terms of the geometry of an $M$-dimensional complex vector space in which the eigenvalues of the covariance matrix of the samples play an essential role.

Another important contribution for spatial signal processing is found in the literature [3]. The authors present an efficient algorithm for ML estimation of the DoA considering multiple emission sources and signals captured by the elements of an antenna array. The estimator can be applied to signals that arrive through multipath propagation. The algorithm is based on an iterative technique referred to as alternating projection (AP), which transforms the nonlinear multivariate maximization problem in a set of unidimensional problems which are easier to simplify. In spite of the convergence achieved for a wide set of simulations, the authors did not assure the convergence for a general problem.

The Estimation of Signal Parameters Via Rotational Invariance Techniques (ESPRIT) algorithm was presented in 
[4]. Although ESPRIT has been used in a scenario of angle of arrival (AoA) estimation, it can be applied to a wide range of problems, including detection and estimation of parameters of sinusoidal signals in the presence of noise. The technique uses the rotational invariance of signal subspaces, as a consequence of the translational invariance of elements in antenna arrays.

Since 1997, the paper of Godora is considered as a reference in spatial signal processing [1]. The paper presents a detailed and broaden treatment of different schemes to adjust a radiated beam and a variety of adaptive algorithms to process signals in arrays.

In [5] another method for DoA estimation from the samples captured by antenna arrays was presented. The method is based on ESPRIT in conjunction with some algorithms for ML estimation of DoA in array signal processing applications. A new, simple, and computationally efficient approach was introduced. It consists of maximizing the ML function over a set of points (a grid) obtained from the sampled data in the array. The technique, referred to as estimation by data-supported grid search, which has roots in the linear regression statistical literature, presents a performance that is similar to the use of genetic algorithms, with a significantly lower computational cost.

A method for DoA estimation based on the support vector machine (SVM) was presented in [6]. In the paper a multiresolution approach for real-time AoA estimation of multiple signals reaching a planar array was introduced. The method is based on a support vector classifier which uses multiscaling to improve the angular resolution of the signal detection process in the region of incidence of electromagnetic waves. Data obtained from the antenna arrays are iteratively transformed by a customized SVM. As a result, a map of probabilities that a signal arrives at the antenna array from a fixed angular direction is determined.

Besides DoA, another important information in the study of processing techniques of signals captured by antenna arrays is the spatial correlation between the elements. Examples of application of the spatial correlation coefficients and the covariance matrix are presented in $[7,8]$. In the first paper, the covariance matrix is used to evaluate the bit error probability of a compact receiver, with maximum ratio combining, under Nakagami fading. In the second paper, the effect of the mutual coupling between randomly located array elements on the performance of an adaptive antenna array (AAA) is investigated.

With the advance of MIMO systems, one can observe a growing need to assess the performance of compact receivers, in which the signal samples at the elements of the array are correlated. In this scenario, it is necessary to characterize the random nature of the directions of arrival using an appropriate probability distribution.

The uniform distribution was widely used to model the DoA. Simplicity is the first reason for its popularity. The second one is the assumption of electromagnetic diffusion isotropy, which can be observed, for instance, in the work of Clarke [9], which assumes uniform distribution for characterizing the DoA of signals that reach the base station, considering multipath. In environments where the electro- magnetic diffusion is not isotropic the uniform distribution may not be applied.

That characteristic of the environment changes the autocorrelation function, as well as the power spectrum of the complex envelope of the signal that is captured by the mobile receiver. Alternative distributions, such as raised cosine, Gaussian, and Laplacian, have been proposed in [1014], and distributions based on geometric models of the channel have also been proposed in $[15,16]$.

Besides the aforementioned models, the Von Mises distribution has received great attention in the context of spatial signal processing. It was firstly proposed to model the nonisotropic propagation mechanism in [17], and spacetime correlation functions were presented. The expressions obtained for the spatial correlation coefficients with Von Mises distribution can be written in terms of Bessel and trigonometric functions, while the correlation coefficients for the Gaussian distribution are written in terms of the complementary error function, with complex parameters [18].

In [19], the authors characterize the nonisotropic DoA using the Von Mises distribution and present functions for the time correlation and power spectral density of the received signals. In [20], this distribution is applied to the computation of the space-time correlation of narrowband multiple-input multiple-output (MIMO) channels subject to Rayleigh fading for a three-dimensional spreading around the mobile station. The authors verified that the nonisotropic diffusion process increases the correlation level as a function of the spacing between the elements of the mobile station antenna.

In [21] the Von Mises distribution is used to determine expressions for the spatial correlation functions of a uniform circular antenna array considering three-dimensional multipath propagation. This distribution is also used in [22] to compute the design parameters of an antenna array in terms of the $\kappa$ adjust parameter.

In this paper, the spatial correlation coefficients of linear and circular antenna arrays are obtained using the Von Mises distribution. The mathematical expressions derived are compared to those obtained using the raised cosine and Gaussian distributions. It is shown that the Von Mises distribution provides expressions that are less complex and leads to similar numerical results when compared to the ones obtained with the raised cosine and Gaussian distributions. To the best of the authors' knowledge, the mathematical expressions of spatial correlation for an arbitrary Q-power cosine distribution for linear array are new. An additional contribution of this paper is the derivation of expressions for the spatial correlation of linear and circular array considering the Von Mises distribution with and without mutual coupling.

\section{The Von Mises Distribution}

The Von Mises distribution is a particular case of the Von Mises-Fisher probability distribution in a $p$-dimensional 
sphere in $\mathbb{R}^{p}$, for $p=2$. The probability density function (pdf), for a unit vector $\mathbf{x}$ of dimension $p$ is given by

$$
p_{p}(\mathbf{x} ; \mu, \kappa)=C_{p}(\kappa) e^{\kappa \mu^{T} \mathbf{x}},
$$

in which $\kappa \geq 0,\|\boldsymbol{\mu}\|=1, T$ is the matrix transposition operator, and $C_{p}(\kappa)$ is a normalization constant:

$$
C_{p}(\kappa)=\frac{\kappa^{p /(2-1)}}{(2 \pi)^{p / 2} \mathrm{I}_{p /(2-1)}(\kappa)},
$$

in which $\mathrm{I}_{v}(x)$ represents the modified Bessel function of the first kind and order $v$. The parameters $\mu$ and $\kappa$ represent the average direction and the accumulation of the distribution. An increase in the value of $\kappa$ implies a concentration of mass around the mean $\mu$.

The distribution was presented by the German physicist Richard Von Mises, in a paper published in 1918 [23], to model differences between the theoretical and measured atomic weighs. If $\phi$ represents the arrival angle of a component that results from the multipath propagation in an urban environment, then the Von Mises distribution is used to model the random variable $\Phi$, whose pdf is given by

$$
p_{\Phi}(\phi)= \begin{cases}\frac{e^{\kappa \cos \left(\phi-\phi_{p}\right)}}{2 \pi \mathrm{I}_{0}(\kappa)}, & -\pi+\phi_{p} \leq \phi \leq \pi+\phi_{p}, \\ 0, & \text { otherwise, }\end{cases}
$$

in which $\phi_{p}$ represents the average direction of a set of directional components and varies in the interval $[0, \pi)$. Plots of $p_{\Phi}(\phi)$ for several values of $\kappa$ are shown in Figure 1. It can be seen that the Von Mises distribution converges to a normal one $\mathcal{N}\left(\phi_{p}, 1 / \kappa\right)$ when $\kappa$ increases, that is,

$$
\lim _{\kappa \rightarrow \infty} p_{\Phi}(\phi)=\frac{e^{-\left(\phi-\phi_{p}\right)^{2} / 2 \sigma^{2}}}{\sigma \sqrt{2 \pi}}, \quad \sigma^{2}=\frac{1}{\mathcal{\kappa}} .
$$

When $\kappa$ goes to zero, the Von Mises distribution converges to the uniform distribution $\mathrm{U}(\phi)$, that is,

$$
\lim _{\kappa \rightarrow 0} p_{\Phi}(\phi)=\mathrm{U}(\phi) .
$$

The $i$ th moment of the random variable $\phi$ is obtained by computing the expected value of $e^{j k \phi}$,

$$
m_{i}=\frac{1}{2 \pi \mathrm{I}_{0}(\kappa)} \int_{-\pi+\phi_{p}}^{\pi+\phi_{p}} e^{j i \phi} e^{\kappa \cos \left(\phi-\phi_{p}\right)} d \phi .
$$

The integral (6) is calculated substituting $u(\phi)=\phi-\phi_{p}$ and using

$$
e^{z \cos (\phi)}=\mathrm{I}_{0}(z)+2 \sum_{l=1}^{\infty} \mathrm{I}_{l}(z) \cos (l \phi)
$$

which gives the moments of $e^{j i \phi}$ for the Von Mises distribution:

$$
m_{i}=\frac{\mathrm{I}_{1}(\kappa)}{\mathrm{I}_{0}(\kappa)} e^{j i \phi_{p}}
$$

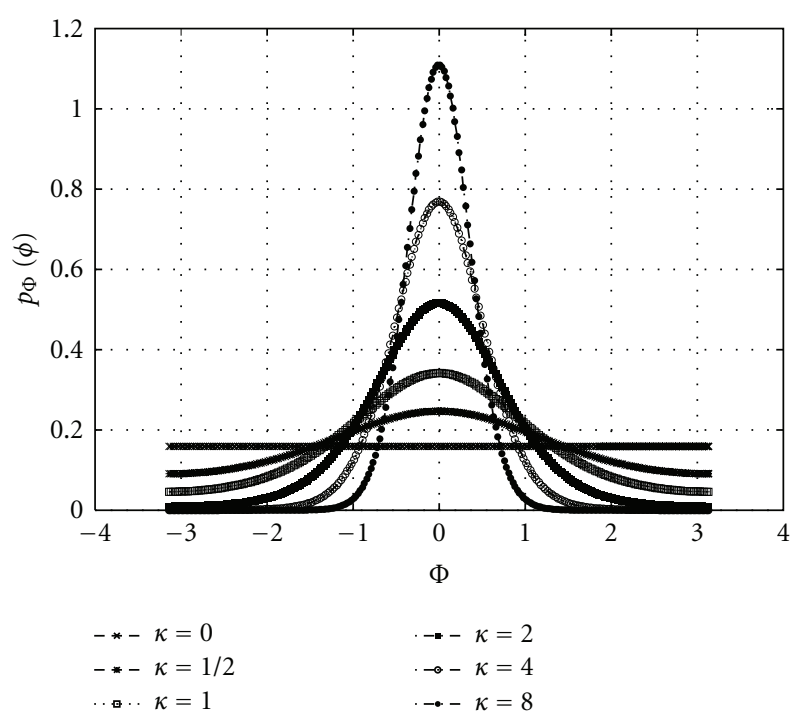

FIgURE 1: Von Mises distribution for several values of $\kappa$.

Other probability distribution functions used to model the directions of arrival in mobile environments are the cosine and Gaussian distributions, whose pdfs are written respectively as

$$
\begin{aligned}
& p_{\Phi}(\phi)= \begin{cases}\frac{k_{c}}{\pi} \cos ^{Q}\left(\phi-\phi_{0}\right), & -\frac{\pi}{2}+\phi_{o} \leq \phi \leq \frac{\pi}{2}+\phi_{o}, \\
0, & \text { otherwise, }\end{cases} \\
& p_{\Phi}(\phi)=\frac{k_{g}}{\sqrt{2 \pi \sigma_{\phi}^{2}}} e^{-\left(\phi-\phi_{0}\right)^{2} / 2 \sigma_{\phi}^{2}}, \quad-\frac{\pi}{2}+\phi_{0} \leq \phi \leq \frac{\pi}{2}+\phi_{o},
\end{aligned}
$$

in which the parameters $k_{c}$ and $k_{g}$ are used to adjust the pdf areas to a unity value, and $Q$ and $\sigma_{\phi}$ control the pdfs shape. The constant $k_{c}$ is given by

$$
k_{c}=\left(\frac{1}{2^{Q}} \sum_{l=0}^{Q}\left(\begin{array}{l}
Q \\
l
\end{array}\right) \operatorname{Sa}\left((2 l-Q) \frac{\pi}{2}\right)\right)^{-1},
$$

in which $\mathrm{Sa}(x)=\sin (x) / x$ is the sample function.

The pdfs for the Von Mises, Gaussian, and Cosine are shown in Figure 2. Note the similarity of the three functions for a proper choice of the parameters $Q, \sigma_{\phi}$, and $\kappa$. This similarity allows the Von Mises distribution to model the DoA in different mobile communications environments.

\section{Computation of the Correlation Coefficients}

To compute the spatial correlation coefficients, consider that the signal samples received by the elements of an equally spaced $N$-element linear array are given by $\mathbf{x}_{l}$, for a separation $d$. On the other hand, the received samples at the 


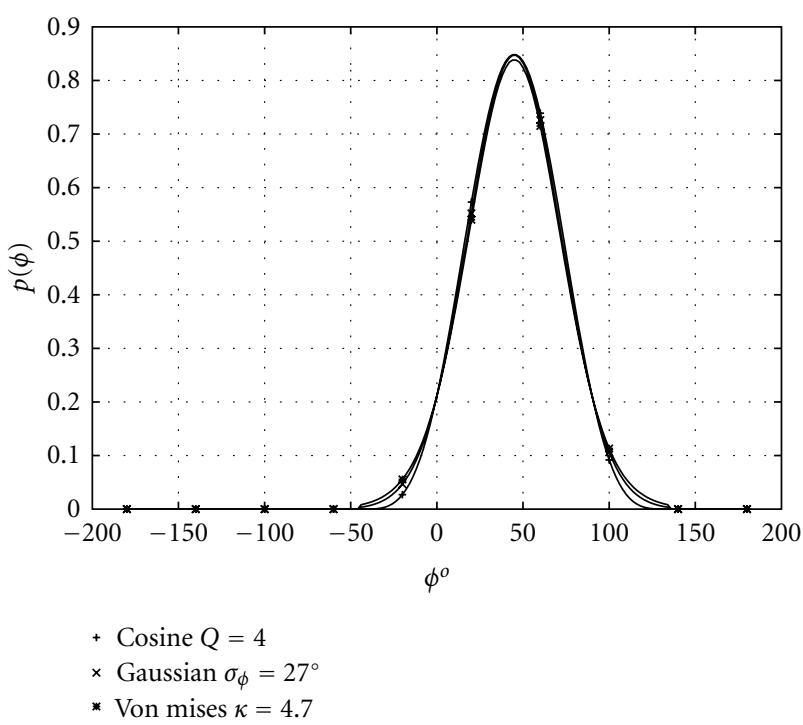

Figure 2: Probability density functions of Von Mises, Gaussian, and cosine for $Q=4, \sigma_{\phi}=27^{\circ}, \kappa=4.7$.

elements of a circular array with radius $a$ are given by $\mathbf{x}_{c}$. In vector form the samples are written as

$$
\begin{gathered}
\mathbf{x}_{l}=\left[\begin{array}{c}
1 \\
e^{-j k d \sin (\phi)} \\
\vdots \\
e^{-j(N-1) k d \sin (\phi)}
\end{array}\right], \\
\mathbf{x}_{c}=\left[\begin{array}{c}
e^{j k a \cos \left(\phi-\tilde{\theta}_{1}\right)} \\
e^{j k a \cos \left(\phi-\tilde{\theta}_{2}\right)} \\
\vdots \\
e^{j k a \cos \left(\phi-\tilde{\theta}_{N}\right)}
\end{array}\right],
\end{gathered}
$$

in which the angles $\tilde{\theta}_{n}$ represent the angular position of the elements for the circular array and $k$ represents the electromagnetic field wave number $2 \pi / \lambda$. For the uniform circular array, $\tilde{\theta}_{n}=2 \pi(n / N)$.

3.1. Computation of the Coefficients for the Linear Array. The spatial correlation coefficients between the samples received by two elements of a linear array with $N$ dipoles, separated by an equal distance $d$, are

$$
\rho(m, n)=E\left[x_{m} x_{n}^{*}\right]
$$

in which $x_{n}^{*}$ is the conjugate of $x_{n}$.
3.1.1. Correlation Coefficients for a Cosine Distribution. For a cosine distribution, the correlation coefficients are given by the integral

$$
\rho_{c}(m, n)=\frac{k_{c}}{\pi} \int_{-\pi / 2+\phi_{o}}^{\pi / 2+\phi_{o}} e^{j(m-n) k d \sin (\phi)} \cos ^{Q}\left(\phi-\phi_{o}\right) d \phi
$$

Using Bessel series and writing $\cos ^{Q}(\phi)$ as a binomial expansion,

$$
\cos ^{Q}(\phi)=\frac{1}{2^{Q}} \sum_{l=0}^{Q}\left(\begin{array}{l}
Q \\
l
\end{array}\right) e^{j(2 l-Q) \phi},
$$

one can obtain the real and imaginary parts of $\rho_{c}(m, n)$, respectively, as

$$
\begin{aligned}
& \Re\left[\rho_{c}(m, n)\right]=\frac{k_{c}}{\pi 2^{Q}} \sum_{l=0}^{Q}\left(\begin{array}{l}
Q \\
l
\end{array}\right) \mathcal{R}_{c}\left((m-n) k d, 2 l-Q, \phi_{o}\right), \\
& \Im\left[\rho_{c}(m, n)\right]=\frac{k_{c}}{\pi 2^{Q}} \sum_{l=0}^{Q}\left(\begin{array}{l}
Q \\
l
\end{array}\right) \ell_{c}\left((m-n) k d, 2 l-Q, \phi_{o}\right),
\end{aligned}
$$

in which the functions $\mathcal{R}_{c}(a, b, c)$ and $\ell_{c}(a, b, c)$ are, respectively, given by (17) presented. Although the integral in (14) can be written in a closed form, a normalization by a constant $k_{c}$ must be carried out and the constant $k_{c}$ depends on the value of $Q$ :

$$
\begin{aligned}
\mathcal{R}_{c}(a, b, c)= & \pi \mathrm{J}_{0}(a) \mathrm{Sa}\left(b \frac{\pi}{2}\right) \\
& +\pi \sum_{i=1}^{\infty} \mathrm{J}_{2 i}(a)\left[\mathrm{Sa}\left((2 i+b) \frac{\pi}{2}\right)+\mathrm{Sa}\left((2 i-b) \frac{\pi}{2}\right)\right] \\
& \times \cos (2 i c) \\
& +\pi \sum_{i=0}^{\infty} \mathrm{J}_{2 i+1}(a)\left[\mathrm{Sa}\left((2 i+1+b) \frac{\pi}{2}\right)\right. \\
& \times \cos ((2 i+1) c), \\
\ell_{c}(a, b, c)= & \pi \sum_{i=1}^{\infty} \mathrm{J}_{2 i}(a)\left[\mathrm{Sa}\left((2 i+b) \frac{\pi}{2}\right)-\mathrm{Sa}\left((2 i-b) \frac{\pi}{2}\right)\right] \\
& \left.\times \sin (2 i c) \quad+\operatorname{Sa}\left((2 i+1-b) \frac{\pi}{2}\right)\right] \\
& +\pi \sum_{i=0}^{\infty} \mathrm{J}_{2 i+1}(a)\left[\mathrm{Sa}\left((2 i+1+b) \frac{\pi}{2}\right)\right. \\
& \times \sin ((2 i+1) c) .
\end{aligned}
$$


3.1.2. Correlation Coefficients for a Gaussian Distribution. For the Gaussian distribution, the spatial correlation function can be written as

$$
\rho_{g}(m, n)=\frac{k_{g}}{\sqrt{2 \pi \sigma_{\phi}^{2}}} \int_{-\pi / 2+\phi_{o}}^{\pi / 2+\phi_{o}} e^{j(m-n) k d \sin \phi} e^{-\left(\phi-\phi_{o}\right)^{2} / 2 \sigma_{\phi}^{2}} d \phi .
$$

The first step in order to solve the integral in (18) is a variable changing. The expansion of the result using Bessel series gives the following expressions for the real and imaginary parts of $\rho_{g}(m, n)$ :

$$
\begin{aligned}
& \mathfrak{R}\left[\rho_{g}(m, n)\right]=\frac{2 k_{g}}{\sqrt{\pi}} \sum_{l=1}^{\infty} \mathrm{J}_{2 l}((m-n) k d) \mathcal{R}_{g}\left(2 l, \phi_{o}, \sigma_{\phi}\right) \\
& +\mathrm{J}_{0}((m-n) k d) \text {, } \\
& \mathfrak{I}\left[\rho_{g}(m, n)\right]=\frac{2 k_{g}}{\sqrt{\pi}} \sum_{l=0}^{\infty} \mathrm{J}_{2 l+1}((m-n) k d) \ell_{g}\left(2 l+1, \phi_{o}, \sigma_{\phi}\right),
\end{aligned}
$$

in which

$$
\begin{gathered}
\mathcal{R}_{g}(a, b, c)=\int_{-\pi / \sqrt{8} c}^{\pi / \sqrt{8} c} \cos (a(\sqrt{2} c u+b)) e^{-u^{2}} d u, \\
\ell_{g}(a, b, c)=\int_{-\pi / \sqrt{8} c}^{\pi / \sqrt{8} \sigma_{\phi} c} \sin (a(\sqrt{2} c u+b)) e^{-u^{2}} d u .
\end{gathered}
$$

Using Euler's identities, the integrals in (20) and (25) can be written in terms of the function $\operatorname{erf}(z)$ as

$$
\begin{aligned}
\mathcal{R}_{g}(a, b, c)= & \frac{\sqrt{\pi}}{2}[\cos (a b) \mathcal{A}(a, c) \\
& -\sin (a b) \mathcal{B}(a, c)] e^{-(a c)^{2} / 2}, \\
\ell_{g}(a, b, c)= & \frac{\sqrt{\pi}}{2}[\sin (a b) \mathcal{A}(a, c)-\cos (a b) \mathcal{B}(a, c)] \\
& \times e^{-(a c)^{2} / 2},
\end{aligned}
$$

in which

$$
\begin{aligned}
\mathcal{A}(a, b)= & \operatorname{Re}\left\{\operatorname{erf}\left(\frac{\pi}{\sqrt{8} b}-j \frac{a b}{\sqrt{2}}\right)\right\} \\
& -\operatorname{Re}\left\{\operatorname{erf}\left(-\frac{\pi}{\sqrt{8} b}-j \frac{a b}{\sqrt{2}}\right)\right\}, \\
\mathcal{B}(a, b)= & \operatorname{Im}\left\{\operatorname{erf}\left(\frac{\pi}{\sqrt{8} b}-j \frac{a b}{\sqrt{2}}\right)\right\} \\
& -\operatorname{Im}\left\{\operatorname{erf}\left(-\frac{\pi}{\sqrt{8} b}-j \frac{a b}{\sqrt{2}}\right)\right\} .
\end{aligned}
$$

The function $\operatorname{erf}(a+j b)$ is defined in [24] as the error function for complex argument and can be calculated using the relations

$$
\begin{gathered}
\operatorname{erf}(x)=1-e^{-x^{2}} w(j x), \\
w(x)=e^{-x^{2}}\left(1+\frac{2 j}{\sqrt{\pi}} \int_{t=0}^{x} e^{-t^{2}} d t\right) .
\end{gathered}
$$

3.1.3. Correlation Coefficients for a Von Mises Distribution. Using the Von Mises distribution, the spatial correlation between two samples of the vector $\mathbf{x}_{l}$ in (11) can be written as

$$
\rho_{\nu}(m, n)=\frac{1}{2 \pi \mathrm{I}_{0}(\kappa)} \int_{-\pi+\phi_{p}}^{\pi+\phi_{p}} e^{j(m-n) k d \sin \phi} e^{\kappa \cos \left(\phi-\phi_{p}\right)} d \phi .
$$

Using Euler's identity and the Bessel series expansion for the real and imaginary parts of $\rho_{v}(m, n)$, it follows that

$$
\begin{aligned}
& \mathfrak{R}\left[\rho_{v}(m, n)\right]=\frac{2}{\mathrm{I}_{0}(\kappa)} \sum_{l=1}^{\infty} \mathrm{J}_{2 l}((m-n) k d) \mathcal{A}_{l}\left(\kappa, \phi_{p}\right) \\
& +\mathrm{J}_{0}((m-n) k d) \\
& \mathfrak{I}\left[\rho_{v}(m, n)\right]=\frac{2}{\mathrm{I}_{0}(\kappa)} \sum_{l=0}^{\infty} \mathrm{J}_{2 l+1}((m-n) k d) \mathscr{B}_{l}\left(\kappa, \phi_{p}\right),
\end{aligned}
$$

in which the auxiliary expressions $\mathcal{A}_{l}(a, b)$ and $\mathscr{B}_{l}(a, b)$ are given by

$$
\begin{aligned}
\mathcal{A}_{l}(a, b) & =\frac{1}{2 \pi} \int_{-\pi+b}^{\pi+b} \cos (2 l \phi) e^{a \cos (\phi-b)} d \phi \\
& =\mathrm{I}_{2 l}(a) \cos (2 l b), \\
\mathcal{B}_{l}(a, b) & =\frac{1}{2 \pi} \int_{-\pi+b}^{\pi+b} \sin ((2 l+1) \phi) e^{a \cos (\phi-b)} d \phi \\
& =\mathrm{I}_{2 l+1}(a) \sin ((2 l+1) b) .
\end{aligned}
$$

From (27), the expressions for the real and imaginary parts of the spatial correlation coefficients for an equally spaced array can be written as

$$
\begin{aligned}
\Re\left[\rho_{v}(m, n)\right]= & 2 \sum_{l=1}^{\infty}\left(\frac{\mathrm{I}_{2 l}(\kappa)}{\mathrm{I}_{0}(\kappa)}\right) \mathrm{J}_{2 l}((m-n) k d) \cos \left(2 l \phi_{p}\right) \\
& +\mathrm{J}_{0}((m-n) k d), \\
\mathfrak{I}\left[\rho_{v}(m, n)\right]= & 2 \sum_{l=0}^{\infty}\left(\frac{\mathrm{I}_{2 l+1}(\kappa)}{\mathrm{I}_{0}(\kappa)}\right) \mathrm{J}_{2 l+1}((m-n) k d) \\
& \cdot \sin \left((2 l+1) \phi_{p}\right) .
\end{aligned}
$$

3.2. Computation of the Coefficients for the Circular Array. The spatial correlation coefficients for the circular array can be computed from the expected value,

$$
\rho_{v}(m, n)=E\left[x_{m} x_{n}^{*}\right]
$$

3.2.1. Correlation Coefficients for a Cosine Distribution. For a cosine distribution, the correlation coefficients of the circular array are given by

$$
\rho_{c}(m, n)=\frac{k_{c}}{\pi} \int_{-\pi / 2+\phi_{o}}^{\pi / 2+\phi_{o}} e^{j k a C_{m, n} \cos \left(\phi-\varphi_{m, n}\right)} \cos ^{Q}\left(\phi-\phi_{o}\right) d \phi .
$$


Writing $\cos ^{Q}(\phi)$ as shown in (15) and using Bessel series for the complex exponential, one can obtain the real and imaginary parts of $\rho_{c}(m, n)$ as

$$
\begin{aligned}
& \mathfrak{R}\left[\rho_{c}(m, n)\right]=\frac{k_{c}}{\pi 2^{Q}} \sum_{l=0}^{Q}\left(\begin{array}{l}
Q \\
l
\end{array}\right) \mathcal{R}_{c}\left(k a C_{m, n}, 2 l-Q, \phi_{o}-\varphi_{m, n}\right), \\
& \Im\left[\rho_{c}(m, n)\right]=\frac{k_{c}}{\pi 2^{Q}} \sum_{l=0}^{Q}\left(\begin{array}{l}
Q \\
l
\end{array}\right) \ell_{c}\left(k a C_{m, n}, 2 l-Q, \phi_{o}-\varphi_{m, n}\right),
\end{aligned}
$$

in which the functions $\mathcal{R}_{c}(a, b, c)$ and $\mathcal{R}_{c}(a, b, c)$ are given by

$$
\begin{aligned}
\mathcal{R}_{c}(a, b, c)= & \pi \mathrm{J}_{0}(a) \mathrm{Sa}\left(b \frac{\pi}{2}\right) \\
+\pi \sum_{l=1}^{\infty}(-1)^{l} \mathrm{~J}_{2 l}(a)[ & \mathrm{Sa}\left((2 l+b) \frac{\pi}{2}\right) \\
& \left.+\mathrm{Sa}\left((2 l-b) \frac{\pi}{2}\right)\right]
\end{aligned}
$$

$\times \cos (2 l c)$

$$
\begin{aligned}
-\pi \sum_{l=0}^{\infty}(-1)^{l} \mathrm{~J}_{2 l+1}(a)[ & \mathrm{Sa}\left((2 l+1+b) \frac{\pi}{2}\right) \\
& \left.-\mathrm{Sa}\left((2 l+1-b) \frac{\pi}{2}\right)\right]
\end{aligned}
$$$$
\times \sin ((2 l+1) c),
$$$$
\begin{aligned}
\ell_{c}(a, b, c)=\pi \sum_{l=1}^{\infty}(-1)^{l} \mathrm{~J}_{2 l}(a)[ & \mathrm{Sa}\left((2 l+b) \frac{\pi}{2}\right) \\
& \left.-\mathrm{Sa}\left((2 l-b) \frac{\pi}{2}\right)\right]
\end{aligned}
$$$$
\times \sin (2 l c)
$$$$
+\pi \sum_{l=0}^{\infty} \mathrm{J}_{2 l+1}(a)\left[\mathrm{Sa}\left((2 l+1+b) \frac{\pi}{2}\right)\right.
$$$$
\left.+\mathrm{Sa}\left((2 l+1-b) \frac{\pi}{2}\right)\right]
$$$$
\times \cos ((2 i+1) c) .
$$

3.2.2. Correlation Coefficients for a Gaussian Distribution. For a Gaussian distribution, the correlation coefficients of the circular array are given by

$$
\rho_{g}(m, n)=\frac{k_{g}}{\sqrt{2 \pi \sigma_{\phi}^{2}}} \int_{-\pi / 2+\phi_{o}}^{\pi / 2+\phi_{o}} e^{j k a C_{m, n} \cos \left(\phi-\varphi_{m, n}\right.} e^{-\left(\phi-\phi_{o}\right)^{2} / 2 \sigma_{\phi}^{2}} d \phi .
$$

The real and imaginary parts of $\rho_{g}(m, n)$ can be calculated using a procedure similar to that used in the linear array.
Applying Bessel expansion to complex exponential one can write $\mathfrak{R}\left[\rho_{g}(m, n)\right]$ and $\mathfrak{I}\left[\rho_{g}(m, n)\right]$ as

$$
\begin{aligned}
& \mathfrak{R}\left[\rho_{g}(m, n)\right]=\frac{2 k_{g}}{\sqrt{\pi}} \sum_{l=1}^{\infty}(-1)^{l} J_{2 l}\left(k a C_{m, n}\right) \\
& \times \mathcal{R}_{g}\left(2 l, \phi_{o}-\varphi_{m, n}, \sigma_{\phi}\right)+\mathrm{J}_{0}\left(k a C_{m, n}\right), \\
& \Im\left[\rho_{g}(m, n)\right]=\frac{2 k_{g}}{\sqrt{\pi}} \sum_{l=0}^{\infty}(-1)^{l} \mathrm{~J}_{2 l+1}\left(k a C_{m, n}\right) \\
& \text { - } \mathcal{R}_{g}\left(2 l+1, \phi_{0}-\varphi_{m, n}, \sigma_{\phi}\right),
\end{aligned}
$$

in which $\mathcal{R}_{g}(a, b, c)$ is given by (21).

3.2.3. Correlation Coefficients for a Von Mises Distribution. Using the Von Mises distribution for two samples of the vector $x_{c}$ in (12), one obtains

$$
\rho_{v}(m, n)=\int_{-\pi+\phi_{p}}^{\pi+\phi_{p}} \frac{e^{j k a C_{m, n} \cos \left(\phi-\varphi_{m, n}\right)}}{2 \pi \mathrm{I}_{0}(\kappa)} e^{\kappa \cos \left(\phi-\phi_{p}\right)} d \phi,
$$

in which

$$
\begin{aligned}
\varphi_{m, n} & =\operatorname{tg}^{-1}\left(\frac{\sin \tilde{\theta}_{m}-\sin \tilde{\theta}_{n}}{\cos \tilde{\theta}_{m}-\cos \tilde{\theta}_{n}}\right), \\
C_{m, n} & =\sqrt{2\left(1-\cos \left(\tilde{\theta}_{m}-\tilde{\theta}_{n}\right)\right)} .
\end{aligned}
$$

Using Euler's identity, one can split the integral (35) into two integrals, which correspond to the real and imaginary parts of $\rho_{v}(m, n)$,

$$
\begin{aligned}
& \mathfrak{R}\left[\rho_{v}(m, n)\right]=\int_{-\pi}^{\pi} \frac{\cos \left(k a C_{m, n} \cos \left(u+\phi_{p}(m, n)\right)\right)}{2 \pi \mathrm{I}_{0}(\kappa)} \\
& \times e^{\kappa \cos (u)} d \phi, \\
& \Im\left[\rho_{v}(m, n)\right]=\int_{-\pi}^{\pi} \frac{\sin \left(k a C_{m, n} \cos \left(u+\phi_{p}(m, n)\right)\right)}{2 \pi \mathrm{I}_{0}(\kappa)} \\
& \times e^{\kappa \cos (u) d \phi,}
\end{aligned}
$$

in which $\phi_{p}(m, n)=\phi_{p}-\varphi_{m, n}$.

Using the Bessel series for $\cos (x \cos \phi)$ and $\sin (x \cos \phi)$, the expression for $\mathfrak{R}\left[\rho_{v}(m, n)\right]$ can be written as

$$
\begin{aligned}
\Re\left[\rho_{v}(m, n)\right]= & 2 \sum_{l=1}^{\infty}(-1)^{l}\left(\frac{\mathrm{I}_{2 l}(\kappa)}{\mathrm{I}_{0}(\kappa)}\right) \mathrm{J}_{2 l}\left(k a C_{m, n}\right) \\
& \cdot \cos \left(2 l \phi_{p}(m, n)\right)+\mathrm{J}_{0}\left(k a C_{m, n}\right) .
\end{aligned}
$$

Following a similar procedure for (38), one can find the imaginary part of the correlation coefficients for a circular array with radius $a$ and equally spaced elements,

$$
\begin{aligned}
\Im\left[\rho_{v}(m, n)\right]= & 2 \sum_{l=0}^{\infty}(-1)^{l}\left(\frac{\mathrm{I}_{2 l+1}(\kappa)}{\mathrm{I}_{0}(\kappa)}\right) \mathrm{J}_{2 l+1}\left(k a C_{m, n}\right) \\
& \cdot \cos \left((2 l+1) \phi_{p}(m, n)\right) .
\end{aligned}
$$


As one can observe from (16), (19), (28), (39), and (40), the spatial correlation coefficients obtained for the Von Mises distribution are simpler and computationally more appropriate than the expressions obtained for the cosine and Gaussian distributions. The expressions for the correlation coefficients for the Von Mises distribution are written only in terms of Bessel functions weighted by trigonometric functions, while the coefficients obtained for the Gaussian distributions are written in terms of Bessel functions weighted by complex error functions. The coefficients expressions for the cosine distribution are obtained using two double summations of Bessel functions weighted by sums of sample functions and trigonometric functions.

\section{Effect of the Mutual Coupling on the Correlation Coefficients}

A radio wave induces an electric current in the element array when it reaches the element. This induced current radiates an electromagnetic field that affects other elements around them. Thus, the received signal in a particular element of the array reflects not only the intensity of the desired signal but also the intensity of signals generated by adjacent elements or other conductive object close to the antenna. This effect, known as mutual coupling, changes the phase distribution of the electric current in the array elements. As a result, gain, bandwidth, radiation pattern, and input impedance of an antenna array are affected $[25,26]$.

The mutual coupling is affected by the separation and the current distribution of the array elements by the wavelength and by the objects located in the near field region. Generally, the most central element of linear and planar arrays are more affected by the coupling [27]. This nonuniform behavior requires individual techniques of impedance matching for each element.

The direction of arrival of the incident wave also affects the coupling. Generally, the direction of arrival and coupling are highly correlated. This occurs more often in arrays that present many phase adjustments. In this case, there is an unbalance among the power of the elements of the array and a consequent changing in coupling between the elements [28].

When the mutual coupling is considered, the dipole length must be taken into account. In this case, the radiated field expressions must be computed for the near field region. The current intensity in each element contributes to the radiated beam, as well as to the distortion of the current distributions in the neighbor elements [29].

To calculate the linear array spatial correlation coefficients, subject to the coupling effect, it is necessary to establish the relation between the voltage vector $\mathbf{V}$, obtained at the array dipoles, and the signal sample vector $\mathbf{S}$, without coupling [30]. This relation is [7]

$$
\mathbf{V}=\mathbf{Z}^{-1} \mathbf{S}
$$

in which $\mathbf{Z}$ and $\mathbf{S}$ are given by

$$
\begin{gathered}
\mathbf{Z}=\left[\begin{array}{ccccc}
1+\frac{Z_{11}}{Z_{L}} & \frac{Z_{12}}{Z_{L}} & \frac{Z_{13}}{Z_{L}} & \cdots & \frac{Z_{1 M}}{Z_{L}} \\
\frac{Z_{21}}{Z_{L}} & 1+\frac{Z_{22}}{Z_{L}} & \frac{Z_{23}}{Z_{L}} & \cdots & \frac{Z_{2 M}}{Z_{L}} \\
\vdots & \vdots & \vdots & \ddots & \vdots \\
\frac{Z_{M 1}}{Z_{L}} & \frac{Z_{M 2}}{Z_{L}} & \frac{Z_{M 3}}{Z_{L}} & \cdots & 1+\frac{Z_{M M}}{Z_{L}}
\end{array}\right], \\
\mathbf{S}=\left[\begin{array}{c}
e^{j 0 k d \sin \phi} \\
e^{j 1 k d \sin \phi} \\
\vdots \\
e^{j(M-1) k d \sin \phi}
\end{array}\right] .
\end{gathered}
$$

It is important to point out that the definition of the mutual impedance matrix in (42) is accurate only for transmitting antennas. For receiving antenna arrays, such as the ones considered for DoA application in this paper, the mutual coupling effect characterized by the matrix in (42) is not accurate because the mutual impedance elements are calculated based on the current distributions of transmitting antenna elements with excitations being at the antenna ports. A more accurate modeling of the antenna mutual impedances, the so-called "receiving mutual impedance", can be found in [31-33]

However, (42) can be used if the receiving antenna arrays for DoA application are excited by electromagnetic waves coming from a short distance (e.g., indoor transmissions), then the current distribution will be different for each of the antenna element.

In the matrix $M \times M$ in (42), the elements $Z_{\mathrm{m} n}$ represent the self-impedance of the $m$ th dipole when $m=n$ and the mutual impedance between the $m$ th dipole and the $n$-dipole when $m \neq n$. Considering that the impedance matrix $\mathbf{Z}^{-1}$ (inverse of $\mathbf{Z}$ ) is given by

$$
\mathbf{Z}^{-1}=\left[\begin{array}{cccc}
a_{11} & a_{12} & \cdots & a_{1 M} \\
a_{21} & a_{22} & \cdots & a_{2 M} \\
\vdots & \vdots & \ddots & \vdots \\
a_{M 1} & a_{M 2} & \cdots & a_{M M}
\end{array}\right],
$$

the voltage vector is

$$
\mathbf{V}=\left[\begin{array}{c}
\sum_{i=1}^{M} a_{1 i} e^{j(i-1) k d \sin \phi} \\
\sum_{i=1}^{M} a_{2 i} e^{j(i-1) k d \sin \phi} \\
\vdots \\
\sum_{i=1}^{M} a_{M i} e^{j(i-1) k d \sin \phi}
\end{array}\right] .
$$


If one takes two samples $r_{m}$ and $r_{n}$ of the voltage vector $\mathrm{V}$, the square of the correlation coefficient modulus can be written as

$$
\left|\rho_{m n}\right|^{2}=\frac{1}{P_{m} P_{n}} \int r_{m} r_{n}^{*} p(\phi) d \phi
$$

in which $P_{m}$ represents the average power of a component from the received signal at the $m$ th antenna array element. For an antenna with $M$ elements, it is given by

$$
P_{m}=\int\left|r_{m}\right|^{2} p(\phi) d \phi, \quad m=1,2, \ldots, M
$$

Substituting one of the vector samples $\mathbf{V}$ into (47), it follows that

$$
\begin{aligned}
P_{m} & =\int_{-\pi+\phi_{p}}^{\pi+\phi_{p}}\left|\sum_{n=1}^{M} a_{m n} e^{j(n-1) k d \sin \phi}\right|^{2} p(\phi) d \phi \\
& =\int_{-\pi+\phi_{p}}^{\pi+\phi_{p}} \sum_{i=1}^{M} \sum_{l=1}^{M} a_{m i} a_{m l}^{*} e^{j(i-l) k d \sin \phi} p(\phi) d \phi,
\end{aligned}
$$

in which $m=1,2, \ldots, M$. that

Applying the Von Mises distribution in (48), it follows

$$
\begin{aligned}
P_{m}= & \sum_{\substack{i=1 \\
i>l}}^{M} \sum_{l=1}^{M} a_{m i} a_{m l}^{*} l\left((i-l) k d, \phi_{p}, \kappa\right) \\
& +\sum_{\substack{i=1 \\
i<l}}^{M} \sum_{l=1}^{M} a_{m i} a_{m l}^{*} l\left((i-l) k d, \phi_{p}, \kappa\right) \\
& +\sum_{i=1}^{M}\left|a_{m i}\right|^{2},
\end{aligned}
$$

in which

$$
\begin{aligned}
\ell\left((i-l) k d, \phi_{p}, \kappa\right) & =\int_{-\pi+\phi_{p}}^{\pi+\phi_{p}} e^{j(i-l) k d \sin \phi} \frac{e^{\kappa \cos \left(\phi-\phi_{p}\right)}}{2 \pi \mathrm{I}_{0}(\kappa)} d \phi \\
& =b_{i l}+j \beta_{i l},
\end{aligned}
$$

with

$$
\begin{aligned}
b_{i l}= & 2 \sum_{n=1}^{\infty}\left(\frac{\mathrm{I}_{2 n}(\kappa)}{\mathrm{I}_{0}(\kappa)}\right) \mathrm{J}_{2 n}((i-l) k d) \cos \left(2 n \phi_{p}\right) \\
& +\mathrm{J}_{0}((i-l) k d), \\
\beta_{i l}= & 2 \sum_{n=0}^{\infty}\left(\frac{\mathrm{I}_{2 n+1}(\kappa)}{\mathrm{I}_{0}(\kappa)}\right) \mathrm{J}_{2 n+1}((i-l) k d) \sin \left((2 n+1) \phi_{p}\right) .
\end{aligned}
$$

Substituting the result from (50) into (49),

$$
\begin{aligned}
P_{m}= & \left(\sum_{i=1}^{M} \sum_{\substack{l=1 \\
l<i}}^{M} a_{m i} a_{m l}^{*} b_{i l}+\sum_{i=1}^{M} \sum_{\substack{l=1 \\
l>i}}^{M} a_{m i} a_{m l}^{*} b_{i l}\right) \\
& +j\left(\sum_{\substack { i=1 \\
\begin{subarray}{c}{l=1 \\
l<i{ i = 1 \\
\begin{subarray} { c } { l = 1 \\
l < i } }\end{subarray}}^{M} a_{m i} a_{m l}^{*} \beta_{i l}-\sum_{i=1}^{M} \sum_{\substack{l=1 \\
l>i}}^{M} a_{m i} a_{m l}^{*} \beta_{i l}\right) \\
& +\sum_{i=1}^{M}\left|a_{m i}\right|^{2} .
\end{aligned}
$$

From (52) one observes that when $i<l$ the terms $a_{m i} a_{m l}^{*}$ and $a_{m i}^{*} a_{m l}$ are complex conjugates. Therefore,

$$
\begin{aligned}
P_{m}= & 2 \sum_{i=1}^{M} \sum_{\substack{l=1 \\
l>i}}^{M} \mathfrak{R}\left\{a_{m i} a_{m l}^{*}\right\} b_{i l}+\sum_{i=1}^{M}\left|a_{m i}\right|^{2} \\
& +2 \sum_{i=1}^{M} \sum_{\substack{l=1 \\
l>i}}^{M} \mathfrak{I}\left\{a_{m i} a_{m l}^{*}\right\} \beta_{i l} .
\end{aligned}
$$

A similar expression can be obtained for the power $P_{n}$. Using (46), the integral $\int r_{m} r_{n}^{*} p(\phi) d \phi$ can be calculated as

$$
\int r_{i}(\phi) r_{l}^{*}(\phi) p(\phi) d \phi=\sum_{n=1}^{M} \sum_{m=1}^{M} a_{i n} a_{l m}^{*}\left(b_{n m}+j \beta_{n m}\right),
$$

in which $b_{n m}+j \beta_{n m}$ can be computed using (51). Therefore, the spatial correlation coefficients are calculated as

$$
\left|\rho_{n m}\right|^{2}=\frac{1}{P_{n} P_{m}}\left|\sum_{i=1}^{M} \sum_{l=1}^{M} a_{n \mathrm{i}} a_{m l}^{*}\left(b_{i l}+j \beta_{i l}\right)\right|^{2} .
$$

For the case of linear dipole elements of length $l$, with $l=n \lambda / 2, n=1,3,5, \ldots$, aligned side by side and centrally fed, the real and imaginary parts of the mutual impedance between two dipoles, referred to as Dipole 1 and Dipole 2, can be obtained using the Electromagnetic Field (EMF) method and can be written as [34]

$$
\begin{aligned}
& R_{12}=\frac{\eta_{0}}{4 \pi}\left[2 \operatorname{Ci}\left(u_{0}\right)-\operatorname{Ci}\left(u_{1}\right)-\operatorname{Ci}\left(u_{2}\right)\right], \\
& X_{12}=-\frac{\eta_{0}}{4 \pi}\left[2 \operatorname{Si}\left(u_{0}\right)-\operatorname{Si}\left(u_{1}\right)-\operatorname{Si}\left(u_{2}\right)\right],
\end{aligned}
$$

in which

$$
\begin{gathered}
u_{0}=k d, \\
u_{1}=k\left(\sqrt{d^{2}+l^{2}}+l\right), \\
u_{2}=k\left(\sqrt{d^{2}+l^{2}}-l\right) .
\end{gathered}
$$

For those equations, $k=2 \pi / \lambda$ is the wave number, $\eta_{0}$ is the medium impedance, approximately $120 \pi \mathrm{ohms}$, and 
$\operatorname{Ci}(x)$ and $\operatorname{Si}(x)$ are the integral sine and cosine functions. Therefore, the impedance between two dipoles $m$ and $n$ is given by $Z_{m n}=R_{m n}+j X_{m n}$, in which

$$
\begin{aligned}
R_{m n}=\frac{\eta_{0}}{4 \pi}[ & 2 \operatorname{Ci}\left(k d_{m n}\right)-\operatorname{Ci}\left(k\left(\sqrt{d_{m n}^{2}+l^{2}}+l\right)\right) \\
- & \left.\operatorname{Ci}\left(k\left(\sqrt{d_{m n}^{2}+l^{2}}-l\right)\right)\right], \\
X_{m n}=-\frac{\eta_{0}}{4 \pi} & {\left[2 \operatorname{Si}\left(k d_{m n}\right)-\operatorname{Si}\left(k\left(\sqrt{d_{m n}^{2}+l^{2}}+l\right)\right)\right.} \\
- & \left.\operatorname{Si}\left(k\left(\sqrt{d_{m n}^{2}+l^{2}}-l\right)\right)\right],
\end{aligned}
$$

with

$$
d_{m n}= \begin{cases}d|m-n|, & \text { for the linear array, } \\
a \sqrt{2\left(1-\cos \left(\tilde{\theta}_{m}-\tilde{\theta}_{n}\right)\right),}, & \text { for the circular array. }\end{cases}
$$

The equation for the computation of the distance between the circular array elements was obtained from the Euclidean distance between two elements located at points $\left(a \cos \tilde{\theta}_{m}, a \sin \tilde{\theta}_{\mathrm{m}}\right)$ and $\left(a \cos \tilde{\theta}_{n}, a \sin \tilde{\theta}_{n}\right)$.

\section{Numerical Evaluation of the Results}

For the numerical evaluation of the expressions presented in this paper, half wavelength linear dipoles were considered with central feeding. For those dipoles, the mutual impedance vectors between the first element, taken as a reference, and the remaining elements are given by (58), and (59). Therefore, the mutual impedances vectors for a linear and a circular array with six elements are given, respectively, by

$$
\begin{array}{r}
\mathbf{Z}_{l}=\left[\begin{array}{c}
1.505667034+j 0.8627287236 \\
0.047354099-j 0.3785965596 \\
-0.062961878+j 0.2041691829 \\
0.052751548-j 0.1363497266 \\
-0.043681351+j 0.1017105490 \\
0.036932473-j 0.0808845309
\end{array}\right], \\
\mathbf{Z}_{c}=\left[\begin{array}{c}
1.505667034+j 0.8627287236 \\
0.047415842-j 0.3811347251 \\
-0.219332270+j 0.1091267749 \\
-0.063208579+j 0.2058290449 \\
-0.219332270+j 0.1091267749 \\
0.047415842-j 0.3811347251
\end{array}\right] .
\end{array}
$$

Both vectors are normalized by the complex conjugate of the dipole self-impedance. The mutual impedance matrices have the form given in (42) and can be obtained from the elements of the vectors $\mathbf{Z}_{l}$ and $\mathbf{Z}_{c}$.
Figures 3 and 4 show plots of the spatial correlation between elements 1 and 3 of the linear array, using the Von Mises distribution to model the AoA. For those curves, $\phi_{p}=$ $30^{\circ}$ and $\phi_{p}=60^{\circ}$ represent the average AoA for the signal components.

Note that in Figure 3(a) and Figure 4(a) the elements 1 and 3 are more correlated for an angle $\phi_{p}=60^{\circ}$ than for an angle $\phi_{p}=30^{\circ}$. This shows the dependence between the correlation and the electromagnetic wave direction of arrival. Furthermore, for the same angle of arrival, there is a dependence between the spatial correlation and the parameter $\kappa$.

Considering Figures 3(b) and 4(b), one can note that increasing values of $\kappa$ corresponds to a highly concentrated beam of electromagnetic waves that arrive at the array and control the degree of correlation. This beam can be produced in anisotropic propagation environments where the reflected components of the electromagnetic waves are added in the direction of the antenna array. This concentration of the beam increases the amplitude of the induced current in some elements of the array and radiate with more intensity to the neighbor elements. Hence, the effect of coupling is increased. This behavior can also be seen in Figure 5(b) for the circular array. The circular distribution of the elements contributes to increasing the correlation because the elements that receive the radiated beam have a direct view of all other elements. In linear arrays the central elements do not have a direct view of the end elements.

Figures 5(a) and 5(b) show different plots of the spatial correlation, for a circular array, as a function of $a / \lambda$, for a six element array. The curves were obtained for different values of $\kappa$ and $\phi_{p}=60^{\circ}$.

From Figure 5(b) one can note that the spatial correlation between elements 1 and 3 can be zero at $a / \lambda \approx 1 / 2$, even for $\kappa$ equals 4 or 8 . Note that when $\kappa>4$ the Von Mises probability density function can approximate a Gaussian distribution, which is commonly used to model AoA in mobile environments.

For the linear and circular configurations the mutual coupling effect increases the correlation between the samples at the parallel elements. As expected, the coupling is affected by the distance between the dipoles, by the angle of arrival, and by the Von Mises distribution parameter $\kappa$. For $\kappa=8$ the curves present higher correlation values for $d=\lambda / 2$.

The amount and shape of the obstacles around the array also determine the mutual coupling between the elements [35]. When the obstacles are concentrated in a specific point of the spatial region nearby the antenna, electromagnetic waves reflected in this agglomerate of obstacles can be concentrated around a main direction and the beam that reaches the array can become more collimated, depending on the variance of the direction of arrival around the mean direction. A collimated beam of waves reaching the array leads to an increase in the power captured by the elements. As a result, the intensity of the induced currents will be augmented, and the effect of coupling will increase. The parameter that determines how well the Von Mises pdf approximates the Gaussian pdf is $\kappa$. A limiting behavior of the distribution is that it approximates the Gaussian pdf with 


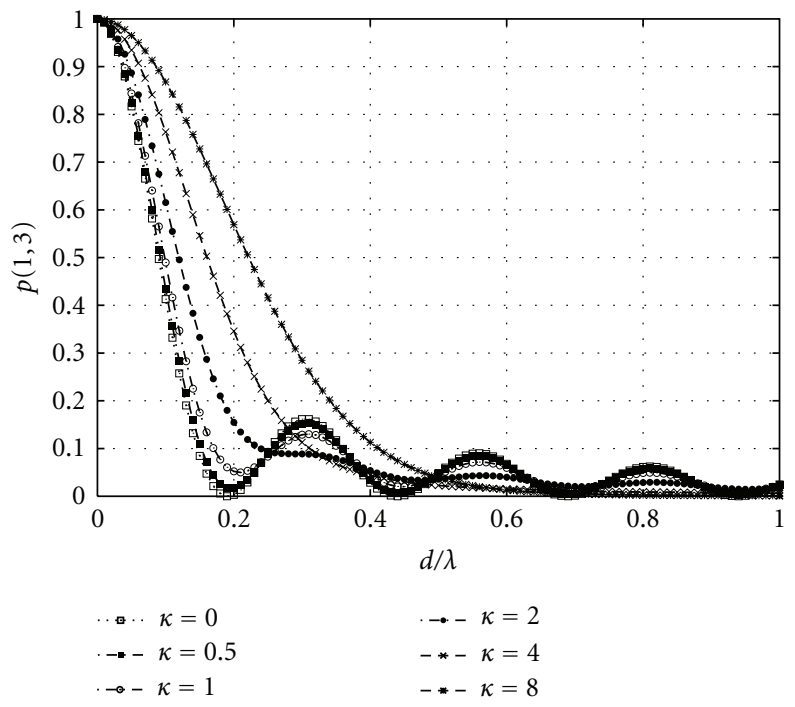

(a) Spatial correlation without mutual coupling

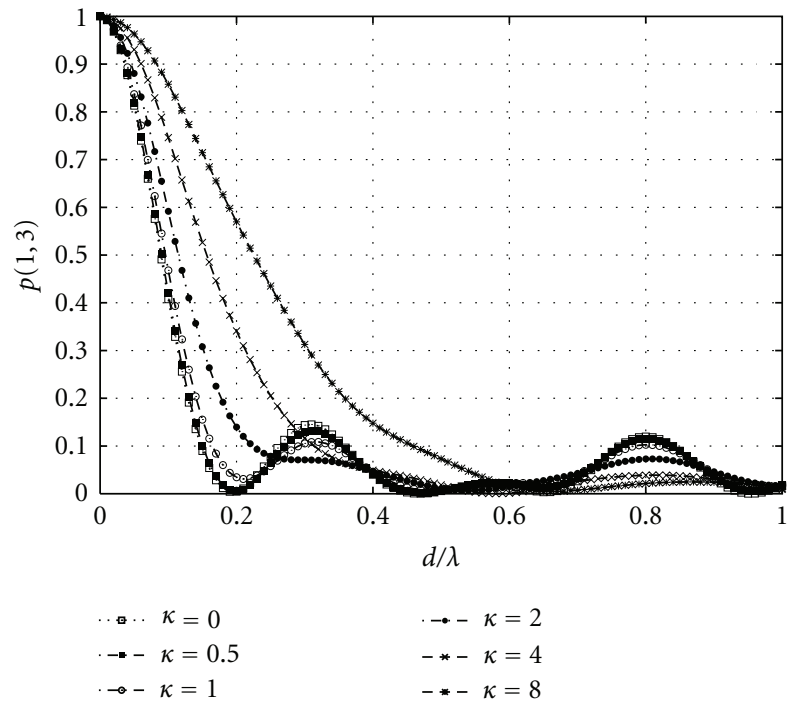

(b) Spatial correlation with mutual coupling

Figure 3: Spatial correlation between elements 1 and 3 for a linear array with six elements, and different values of the parameter $\kappa$, with $\phi_{p}=30^{\circ}$, as a function of $d / \lambda$.

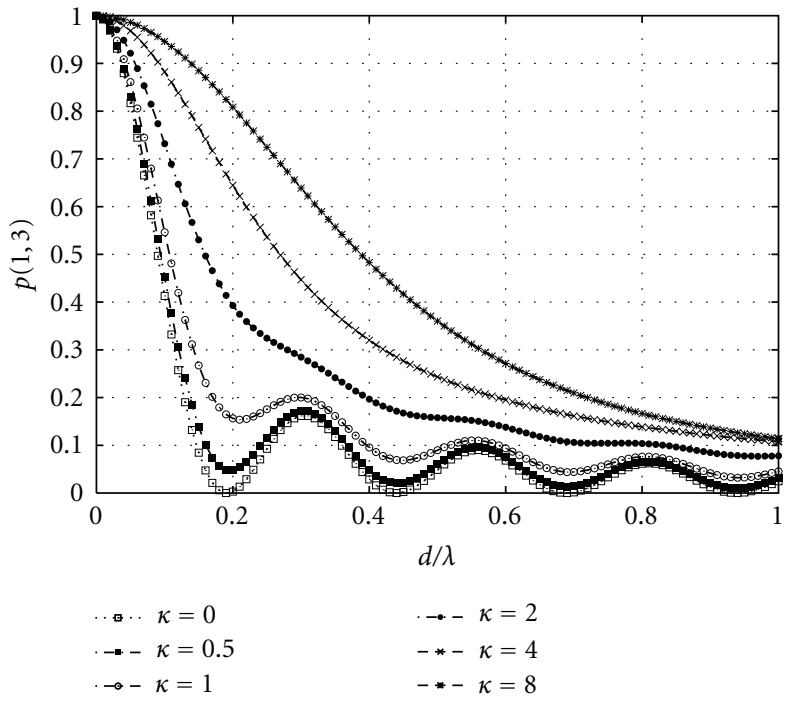

(a) Spatial correlation without mutual coupling

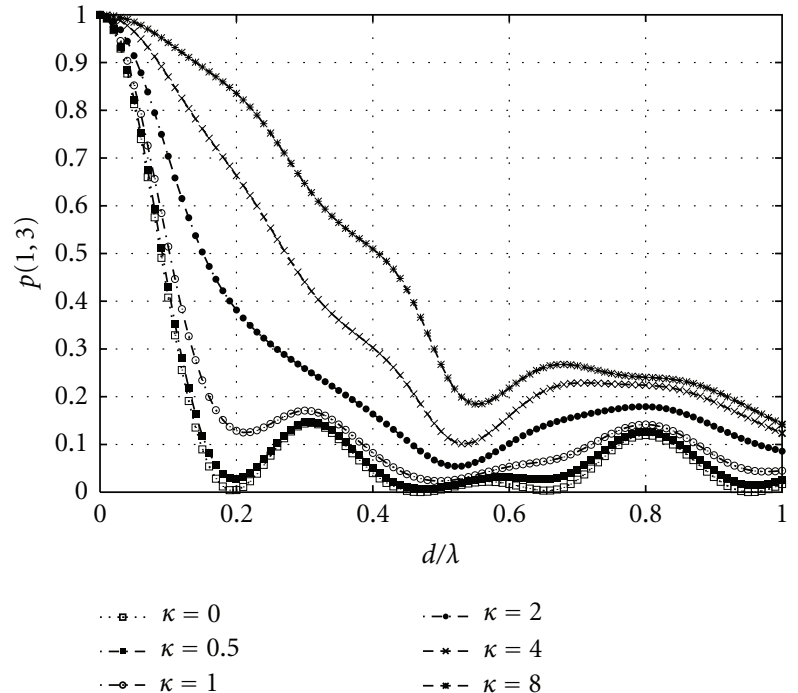

(b) Spatial correlation with mutual coupling

Figure 4: Spatial correlation between elements 1 and 3 for a linear array with six elements, and different values of the parameter $\kappa$, with $\phi_{p}=60^{\circ}$, as a function of $d / \lambda$.

variance $\sigma^{2}=1 / \kappa$. Hence, a high $\kappa$ leads to a small variance for the Gaussian approximation and the beam of waves that reaches the array collimates and the intensity of the distribution of induced currents in the elements increases.

\section{Conclusions}

This paper presented a mathematical comparison between the spatial correlation expressions for cosine, Gaussian, and Von Mises probability distributions, for linear and circular antenna arrays. The expressions obtained for the Von Mises distribution can include or not the mutual coupling effect between the elements and are simpler than those obtained for the cosine and the Gaussian distributions of the angle of arrival. The use of the Von Mises distribution allows the spatial correlation expressions to depend only on Bessel and trigonometric functions. An exact expression for the spatial correlation is also presented, considering the mutual coupling, for the linear and circular arrays and an arbitrary number of half wave dipole elements. From the numerical 


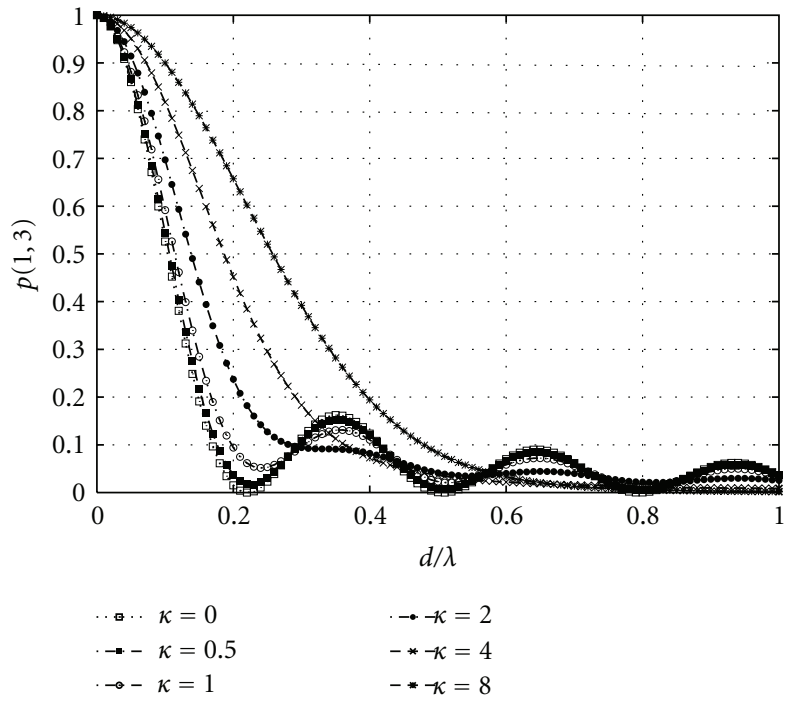

(a) Spatial correlation without mutual coupling

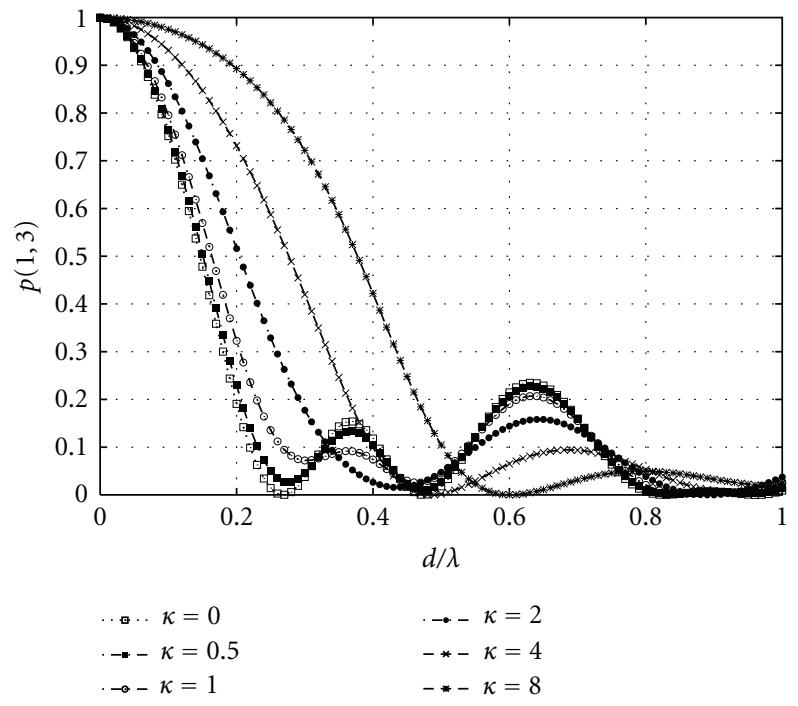

(b) Spatial correlation with mutual coupling

Figure 5: Spatial correlation between elements 1 and 3 for a circular array with six elements, and different values of the parameter $\kappa$, with $\phi_{p}=60^{\circ}$, as a function of $a / \lambda$.

evaluation of the expressions, it is observed that in some cases the separation of $\lambda / 2$ is not sufficient to decorrelate the signal.

\section{References}

[1] L. C. Godara, "Application of antenna arrays to mobile communications, part II: beam-forming and direction-ofarrival considerations," Proceedings of the IEEE, vol. 85, no. 8, pp. 1195-1245, 1997.

[2] R. O. Schmidt, "Multiple emitter location and signal parameter estimation," IEEE Transactions on Antennas and Propagation, vol. AP-34, no. 3, pp. 276-280, 1986.

[3] I. Ziskind and M. Wax, "Maximum likelihood localization of multiple sources by alternating projection," IEEE Transactions on Acoustics, Speech, and Signal Processing, vol. 36, no. 10, pp. 1553-1560, 1988.

[4] R. Roy and T. Kailath, "ESPRIT-estimation of signal parameters via rotational invariance techniques," IEEE Transactions on Acoustics, Speech, and Signal Processing, vol. 37, no. 7, pp. 984-995, 1989.

[5] P. Stoica and A. B. Gershman, "Maximum-likelihood DOA estimation by data-supported grid search," IEEE Signal Processing Letters, vol. 6, no. 10, pp. 273-275, 1999.

[6] M. Donelli, F. Viani, P. Rocca, and A. Massa, "An innovative multiresolution approach for DOA estimation based on a support vector classification," IEEE Transactions on Antennas and Propagation, vol. 57, no. 8, pp. 2279-2292, 2009.

[7] J. Luo, J. R. Zeidler, and S. McLaughlin, "Performance analysis of compact antenna arrays with MRC in correlated Nakagami fading channels," IEEE Transactions on Vehicular Technology, vol. 50, no. 1, pp. 267-277, 2001.

[8] M. J. Lopes Alves and M. S. de Alencar, "A linear adaptive antenna array with random spacing and coupling effects," Journal of Microwaves and Optoelectronics, vol. 7, no. 1, pp. 16$25,2008$.
[9] R. H. Clarke, "A statistical theory of mobile-radio reception," The Bell System Technical Journal, vol. 47, pp. 957-1000, 1968.

[10] J. S. Sadowsky and V. Kafedziski, "On the correlation and scattering functions of the WSSUS channel for mobile communication," IEEE Transactions on Vehicular Technology, vol. 47, pp. 270-282, 1998.

[11] W. R. Braun and U. Dersch, "A physical mobile radio channel model," IEEE Transactions on Vehicular Technology, vol. 40, no. 2, pp. 472-482, 1991.

[12] A. Kuchar, E. Aparicio, J.-P. Rossi, and E. Bonek, "Azimuth, elevation, and delay of signals at mobile station site," in Proceedings of the 8th Virginia Tech Symposium on Wireless Personal Communications, pp. 99-110, Blacksburg, Va, USA, 1998.

[13] W. C. Y. Lee, "Effect on correlation between two mobile radio base-station antennas," IEEE Transactions on Communications, vol. 21, no. 11, pp. 1214-1224, 1973.

[14] F. Adachi, M. T. Feeney, A. G. Williamson, and J. D. Parsons, "Crosscorrelation between the envelopes of $900 \mathrm{MHz}$ signals received at a mobile radio base station site," IEE ProceedingsCommunications, Radio and Signal Processing, vol. 133, no. 6, pp. 506-512, 1986.

[15] P. Petrus, J. H. Reed, and T. S. Rappaport, "Effects of directional antennas at the base station on the doppler spectrum," IEEE Communications Letters, vol. 1, no. 2, pp. 4042, 1997.

[16] J. C. Liberti and T. S. Rappaport, "A geometrically based model for line-of-sight multipath radio channels," in Proceedings of the IEEE Vehicular Technology Conference (VTC '96), pp. 844848, Atlanta, Ga, USA, 1996.

[17] A. Abdi and M. Kaveh, "A versatile spatio-temporal correlation function for mobile fading channels with non-isotropic scattering ," in Proceedings of the 10th IEEE Workshop on Statistical Signal and Array Processing, 2000.

[18] J. Fuhl, A. F. Molisch, and E. Bonek, "Unified channel model for mobile radio systems with smart antennas," IEE 
Proceedings-Radar, Sonar Navigation, vol. 145, no. 1, pp. 32 41, 1998.

[19] A. Abdi, J. A. Barger, and M. Kaveh, "A parametric model for the distribution of the angle of arrival and the associated correlation function and power spectrum at the mobile station," IEEE Transactions on Vehicular Technology, vol. 51, no. 3, pp. 425-434, 2002.

[20] A. A. Al-Kheir, K. A. Qaraqe, and M.-S. Alouini, "Space-time channel correlation of MIMO rayleigh fading based on nonisotropic 3D scattering," in Proceedings of the International Conference on Signal Processing and Communications (ICSPC '07), pp. 648-651, Dubai, United Arab Emirates, November 2007.

[21] K. Mammasis, R. W. Stewart, and J. S. Thompson, "Spatial Fading Correlation model using mixtures of von Mises Fisher distributions," IEEE Transactions on Wireless Communications, vol. 8, no. 4, Article ID 4907468, pp. 2046-2055, 2009.

[22] J. Ren and R. G. Vaughan, "Spaced antenna design in directional scenarios using the von mises distribution," in Proceedings of the 70th IEEE Vehicular Technology Conference (VTC '09), pp. 1-5, Anchorage, Alaska, USA, September 2009.

[23] R. V. Mises, "Über die ganzzahligkeit der atomgewicht and verwandte fragen," Physikal Z, vol. 19, pp. 490-500, 1918.

[24] M. Abramowitz and I. A. Stegun, Handbook of Mathematical Functions, Dover pubications, New York, NY, USA, 1970.

[25] J. W. Wallace and M. A. Jensen, "Mutual coupling in MIMO wireless systems: a rigorous network theory analysis," IEEE Transactions on Wireless Communications, vol. 3, no. 4, pp. 1317-1325, 2004.

[26] J. Wallace, T. Shawcroft, and M. Jensen, "Modeling antenna coupling and correlation in rapidly fading MIMO channels," in Proceedings of the 1st European Conference on Antennas and Propagation (EuCAP '06), Nice, France, November 2006.

[27] H. A. Mehmet, K. Ozdemiz, and E. Arvas, "Mutual coupling effect in multi-antenna wireless," in Proceedings of the IEEE Globecom, San Francisco, Calif, USA, December 2003.

[28] M. A. Jensen and J. W. Wallace, "Analysis of coupling in multi-antenna communication systems," IEICE Transactions on Electronics, vol. E87-C, no. 9, pp. 1418-1424, 2004.

[29] N. W. Bikhazi and M. A. Jensen, "Impact of coupling on multiple-antenna capacity in correlated fast-fading environments," IEEE Transactions on Vehicular Technology, vol. 58, no. 3, pp. 1595-1597, 2009.

[30] J. Wallace, H. Ozcelik, M. Herdin, E. Bonek, and M. Jensen, "Power and complex envelope correlation for modeling measured indoor MIMO channels: a beamforming evaluation," in Proceedings of the 58th IEEE Vehicular Technology Conference (VTC-Fall '03), vol. 1, pp. 363-367, Orlando, Fla, USA, October 2003.

[31] H. T. Hui, "A new definition of mutual impedance for application in dipole receiving antenna arrays," IEEE Antennas and Wireless Propagation Letters, vol. 3, no. 1, pp. 364-367, 2004.

[32] H. T. Hui, "A practical approach to compensate for the mutual coupling effect in an adaptive dipole array," IEEE Transactions on Antennas and Propagation, vol. 52, no. 5, pp. 1262-1269, 2004.

[33] H.-S. Lui, H. T. Hui, and M. S. Leong, "A note on the mutualcoupling problems in transmitting and receiving antenna arrays," IEEE Antennas and Propagation Magazine, vol. 51, no. 5, Article ID 5432083, pp. 171-176, 2009.

[34] C. A. Balanis, Antenna Theory: Analysis and Design, John Wiley \& Sons, 1997.
[35] J. Wallace and M. Jensen, "Impact of antenna coupling on diversity performance: complete network theory analysis," in Proceedings of the IEEE International Conference on Communications (ICC '04), vol. 2, pp. 947-951, Paris, France, June 2004. 

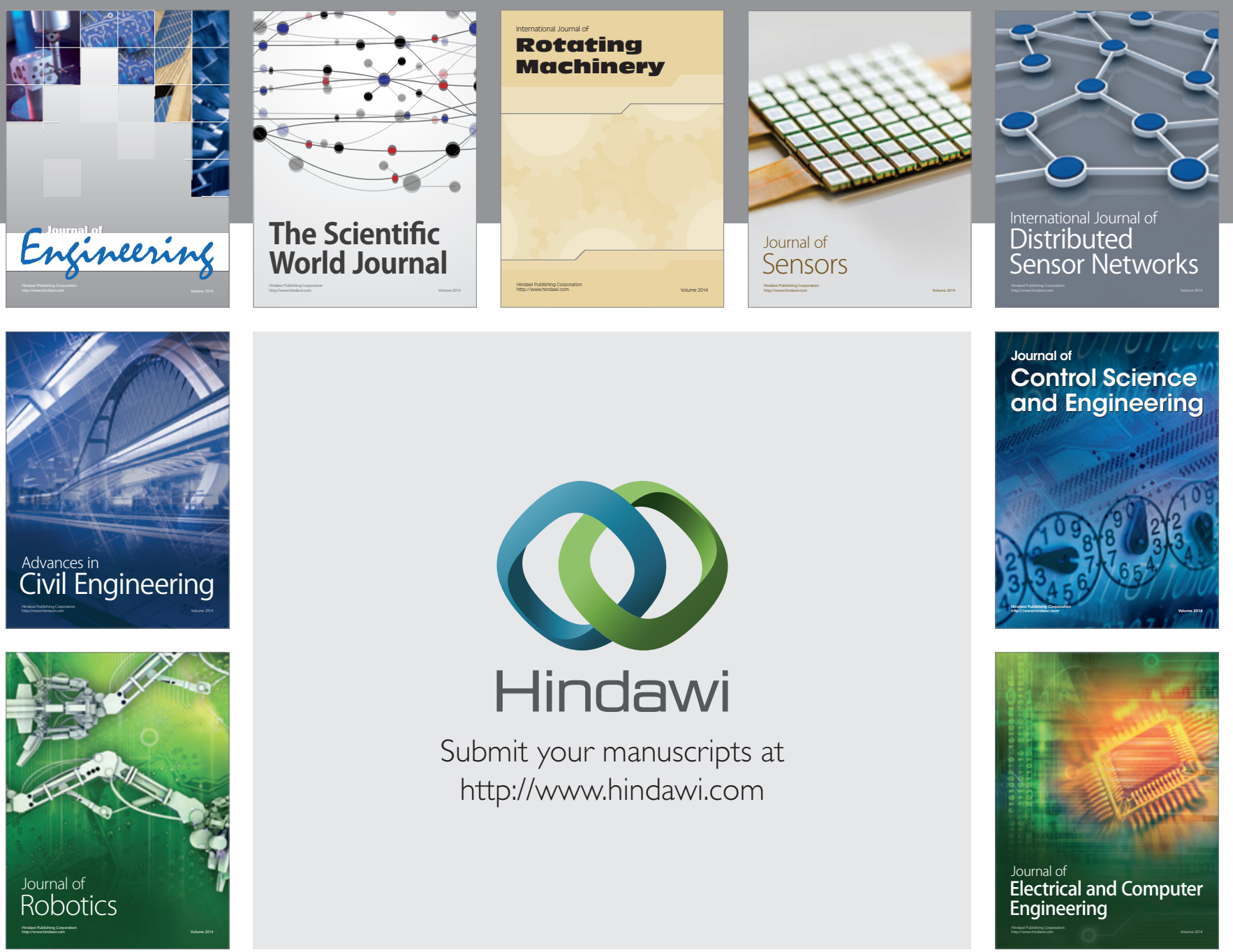

Submit your manuscripts at

http://www.hindawi.com
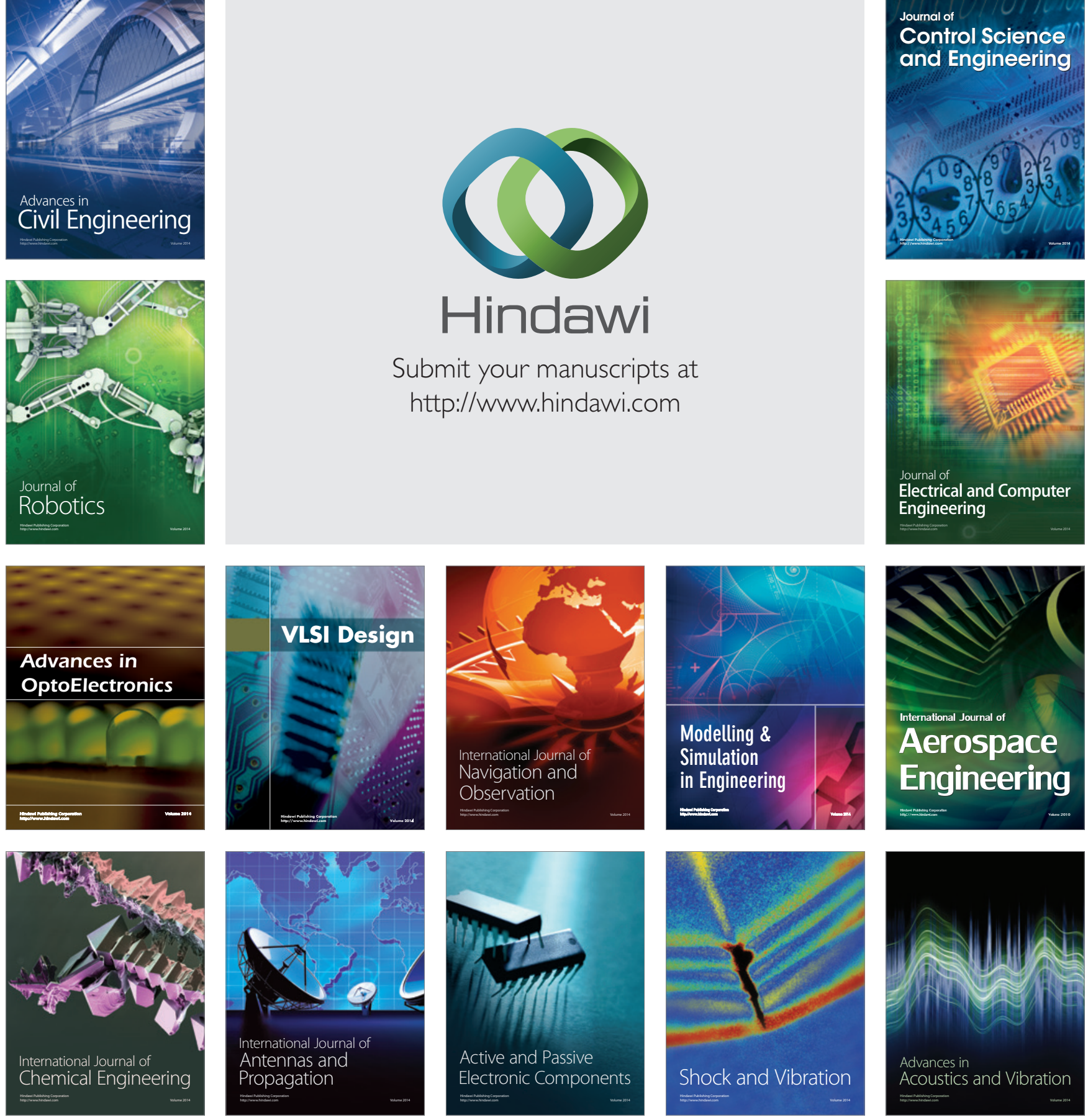